\title{
Modélisation d'une boucle diphasique à pompage capillaire pour utilisation spatiale
}

\author{
The modelling of a two-phase capillary pumped loop for use in space
}

par T. Tjiptahardja, M. Amidieu, B. Moschetti

Aerospatiale Espace et Défense - Cannes

This paper presents the preliminary thermal and hydraulic model of Stentor (Satellite de Télécommunication pour l'Expérimentation de Nouvelles Technologies en orbite) Capillary Pumped Loop (CPL). This multiple-evaporator two-phase ammonia loop acquires, transports and rejects heat dissipated by the Stentor Tx Antenna. The loop consists of six evaporators, six integrated-reservoirs, six capillary links, fluid transport lines and two rectilinear condensers. The validity and limitations of the modelling assumptions are presented and discussed. The CPL elements are built with the component models available in SINDAFFLUINT thermal and fluid software code.

\section{INTRODUCTION}

Le satellite STENTOR (Satellite de Télécommunication pour l'Expérimentation de Nouvelles Technologies en ORbite) est un démonstrateur technologique pour la nouvelle génération de satellite de communication géostationnaire (figure 1). Parmi de nouvelles technologies proposées figurent de nouveaux dispositifs de contrôle thermique basés sur les boucles diphasiques à pompage capillaire.

Une boucle diphasique à pompage capillaire comme la boucle CPL du satellite STENTOR (figure 2) est un dispositif nouveau de contrôle thermique. Il est basé sur l'utilisation d'un milieu poreux qui développe des forces capillaires permettant la circulation du fluide dans la boucle. L'avantage de ce dispositif par rapport à un caloduc est notamment lié à sa capacité de transporter un flux thermique important sur une longue distance.

Un schéma de principe du fonctionnement d'une telle boucle est présenté dans la figure 3 . Lorsqu'un chargement thermique est appliqué sur l'évaporateur, la vapeur est générée à la surface externe de la mèche capillaire en contact avec la paroi d'évaporateur. Elle est ensuite évacuée depuis l'évaporateur vers le condenseur. Le titre thermodynamique de cet écoulement va diminuer le long de la boucle jusqu'à son retour vers l'évaporateur. Plusieurs régimes d'écoulement diphasique sont rencontrés durant cette circulation du fluide. En contact avec une paroi relativement plus froide à sa sortie de l'évaporateur, la vapeur commence à se condenser. Le film liquide formé sur la paroi interne du tube est entraînée par l'écoulement de vapeur (écoulement annulaire). A une certaine position de la boucle, les forces de tension superficielle vont dominer. L'écoulement diphasique va devenir intermittent, constitué de bouchons de liquide mélangés avec des poches de vapeur qui se condensent. La poursuite du processus de condensation de ces poches de vapeur va aboutir à un écoulement dispersé à bulles. Au retour dans le canal d'alimentation en liquide de l'évaporateur, le fluide en contact avec la partie interne de la mèche se trouve dans un état liquide sousrefroidi. Ce liquide est ensuite pompé vers la partie externe

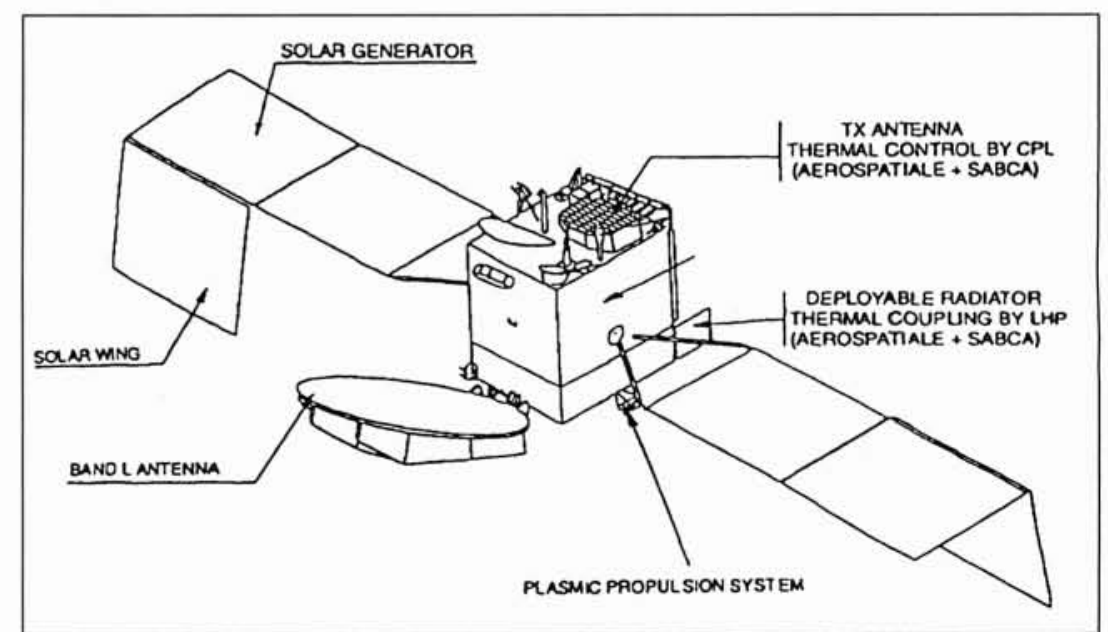

1. Satellite de Télécommunication pour l'Expérimentation de Nouvelles Technologies en ORbite - STENTOR. 
de la mèche d'évaporateur grâce aux forces capillaires développées au sein de la mèche. L'évaporation recommence alors au niveau des ménisques formés au voisinage de la zone de contact entre la mèche capillaire et la paroi interne de l'évaporateur chaud.

\section{II $\square$ BESOINS DE MODÉLISATION}

\subsection{Besoins de modélisation thermohydraulique}

Il existe deux niveaux de besoin de modélisation de la boucle CPL du satellite STENTOR : niveau système (utilisateur de boucle : Aerospatiale Cannes) et niveau composant (fabricant de boucle : SABCA, Belgique). Au niveau composant, les techniques d'analyse bidimensionnelles ou tridimensionnelles sont le plus souvent utilisées. Elles permettent la prédiction du comportement détaillé de chaque composant de la boucle CPL notamment ses performances et ses limitations de fonctionnement. Elles permettent également de valider les modèles de composant de la boucle qui sont utilisés au niveau système. A ce niveau, les techniques d'analyse

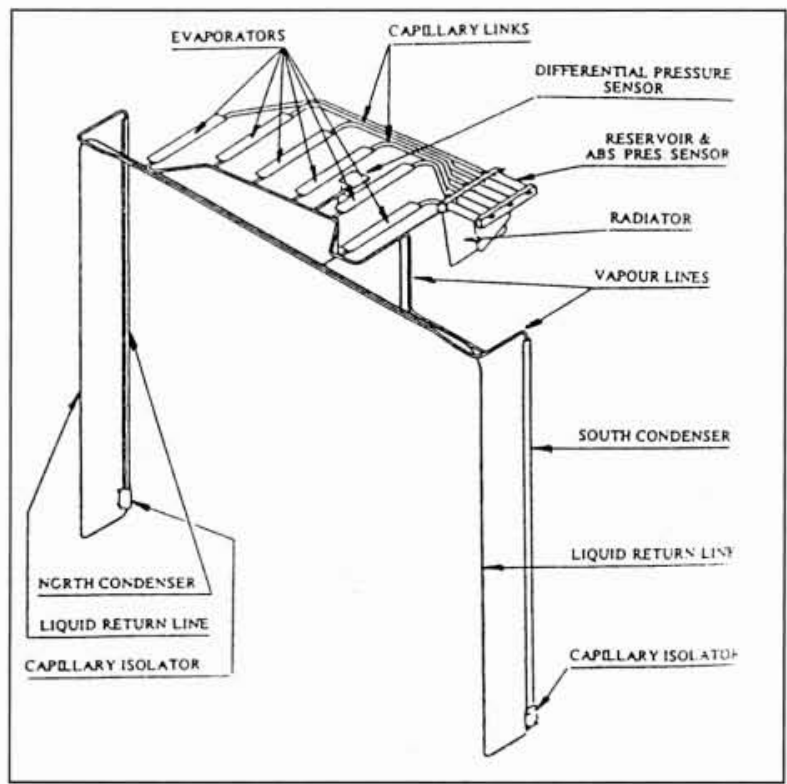

2. Boucle diphasique à pompage capillaire $(\mathrm{CPL}) \mathrm{du}$ satellite STENTOR. utilisées sont historiquement monodimensionnelles. Les modèles de composant de la boucle sont donc simplifiés. L'utilisateur de la boucle CPL doit pouvoir prédire - au sol et en vol orbital - les performances thermohydrauliques et les limitations physiques de la boucle aussi bien en régime permanent qu'en régime transitoire. Les interactions avec les autres composants du satellite et l'environnement doivent être naturellement prises en compte. Pour toutes les techniques d'analyse décrites ci-dessus, les validations expérimentales sont requises. Le modèle de la boucle CPL du satellite STENTOR traite un problème thermohydraulique fortement couplé. Il se place dans le contexte suivant : niveau système (utilisateur de boucle) et approche monodimensionnelle (méthode nodale).

\subsection{Outil d'analyse thermohydraulique}

Une analyse comparative de plusieurs codes a été effectuée par Aerospatiale Cannes en vue de répondre aux besoins de modélisation thermohydraulique de deux boucles diphasiques du satellite STENTOR [1]. La construction du modèle thermohydraulique de la boucle CPL du satellite STENTOR s'effectue à l'aide du code SINDA/FLUINT et SinapsPlus [2]. SINDA/FLUINT ('Systems Improved Numerical Differencing Analyzer / Fluid Integrator', anciennement SINDA '85) est un code numérique destiné à l'analyse des systèmes thermo-fluides qui peuvent être représentés en formulation différences-finies ou en formulation nodale. Le code est capable de modéliser les réseaux fluides monophasiques et diphasiques en régimes permanent et transitoire, leurs 'hardware' correspondants ainsi que leurs processus de transfert de chaleur (conductif, convectif et radiatif) associés. L'origine de SINDA remonte au début des années 60 . Il utilise l'approche réseau thermique : un problème est découpé en nœuds (conservation d'énergie) et conducteurs (à travers lesquels l'énergie est échangée en mode conductif et radiatif). FLUINT remonte - quant à lui - au milieu des années 80. Il introduit les notions de 'lumps' (nœuds fluides) et 'paths' (conducteurs fluides) qui sont en analogie directe avec les nœuds et conducteurs thermiques classiques. Il introduit en outre des éléments appelés 'ties' qui n'ont pas d'équivalent dans SINDA. Ces éléments permettent l'échange de chaleur entre les nœuds thermiques et les nœuds fluides. En résumé, contrairement aux modèles

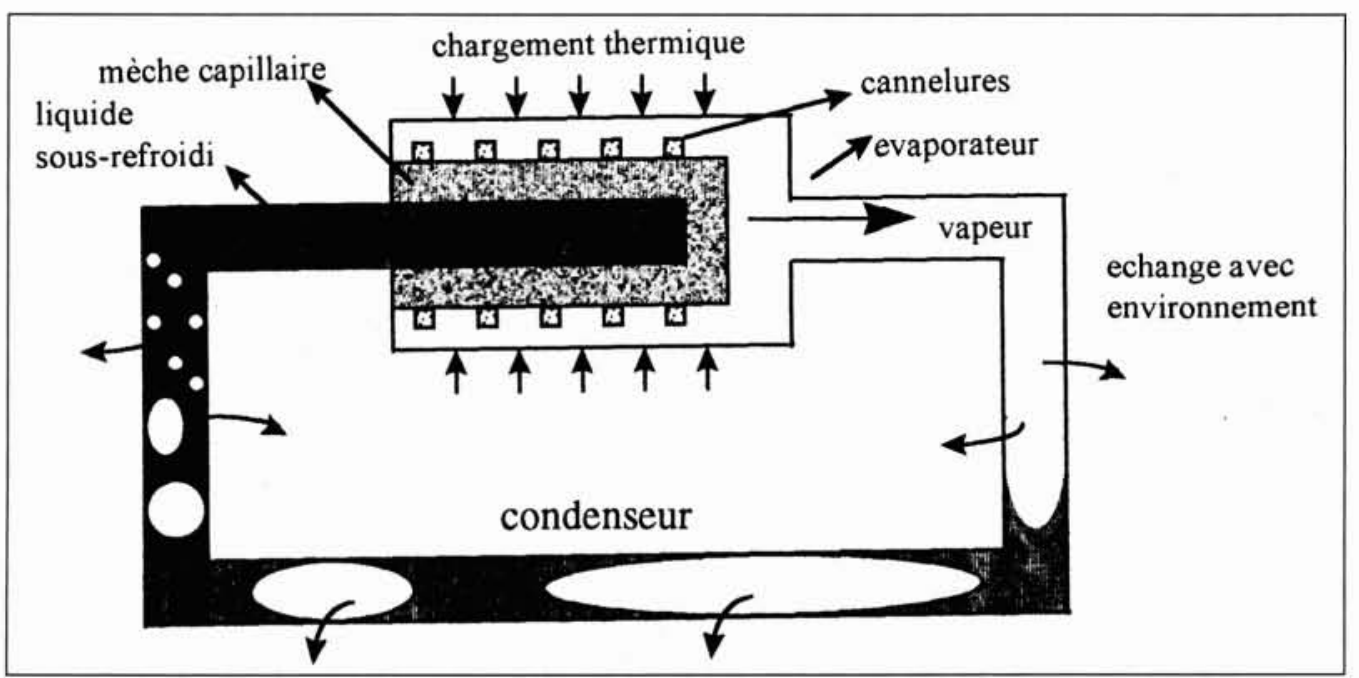

3. Schéma de principe de fonctionnement d'une boucle diphasique à pompage capillaire. 
réseaux thermiques classiques qui ne traitent que le bilan d'énergie, les modèles réseaux fluides traitent de plus les bilans de masse et de quantité de mouvement. SinapsPlus ('SINDA Application Programming System') est le pré- et post - processeur graphique du code SINDA/FLUINT.

\section{III $\square$ DÉCOUPAGE NODAL ET DISCUSSIONS SUR LES MODÈLES DE COMPOSANT}

Les figures 4 et 5 donneront un aperçu rapide et global du découpage nodal de la boucle CPL du satellite STENTOR. Dans le modèle thermique LINES figurent les nœuds thermiques et leurs conducteurs représentant la partie métallique de la boucle (paroi d'évaporateur, paroi de réservoir, parois des condenseurs, paroi des lignes de transport de fluide), la partie extérieure de la mèche capillaire de l'évaporateur en condition de saturation (le point de vaporisation du fluide) et les puits thermiques représentant les environnements ambiant et spatial (figure 4). Dans le modèle fluide LOOP figurent les nœuds fluides et leurs conducteurs qui représentent le fluide à l'intérieur de la boucle ainsi que les liens convectifs qui relient ces nœuds fluides aux nœuds thermiques (figure 5).

\subsection{Réservoir}

Les six réservoirs de la boucle CPL ont été conçus pour se comporter de manière homogène en pression et en température en vue de faciliter la gestion des taux de vide à l'intérieur de ces six éléments. Du point de vue de la modélisation, un seul volume de contrôle suffit donc pour traiter le fonctionnement nominal de ces six réservoirs intégrés. Dans les développements qui suivent, il ne sera donc plus question que d'un seul réservoir (sous-entendu six réservoirs homogènes). Au sein du modèle SINDA/FLUINT de la boucle CPL du satellite STENTOR, le fluide contenu dans le réservoir est modélisé par un seul nœud fluide ('TANK') homogène. Le fluide diphasique du réservoir est donc supposé en état d'équilibre thermodynamique et bien mélangé sans aucune variation axiale. La paroi du réservoir, considérée comme uniforme en température, est modélisée elle aussi - par un seul nœud thermique. Le radiateur dédié au réservoir participe aux efforts de compenser le chargement thermique parasite provenant des évaporateurs (voir les discussions sur ce sujet au paragraphe 3.2). Ce radiateur est représenté par un nœud thermique. La répartition de température sur toute la surface du radiateur est donc supposée uniforme.

Le réservoir diphasique de la boucle CPL est de configuration complexe. Une structure capillaire particulière placée à l'intérieur du réservoir gère l'acquisition du fluide et le positionnement du fluide en condition orbitale de microgravité et durant des essais au sol. Ce réservoir échange de la chaleur avec son environnement. En raison de la présence d'un radiateur placé en proximité du réservoir, la paroi du celui-ci est plus froide que le fluide à l'intérieur. De plus, nous devons également tenir compte de la stratification du fluide diphasique due à la gravité terrestre et du positionnement du fluide diphasique sous la condition orbitale en microgravité. La détermination du coefficient de transfert de chaleur entre fluide et paroi interne du réservoir n'est donc pas aisée. A ce stade de l'étude, plusieurs corrélations internes au code SINDA/FLUINT sont testées notamment celle de Rohsenow [3]. La caractérisation plus précise du coefficient de film nécessitera sans doute un recours aux procédures de corrélation à quelques résultats d'essais au sol et en vol.

En n'employant qu'un seul nœud fluide pour modéliser le fluide dans le réservoir, on suppose implicitement que les transferts de chaleur et de masse entre les deux phases sont infinis. Or, la modélisation du déséquilibre thermodynamique entre les deux phases liquide-vapeur d'un réservoir diphasique est sans doute nécessaire. En effet, lorsqu'un liquide relativement froid pénètre le réservoir, la pression de la boucle peut augmenter de façon transitoire ou diminuer (de manière parfois dangereuse pour le fonctionnement de la boucle) selon l'effet prédominant, respectivement, du phénomène de compression ou de condensation de la vapeur dans le réservoir. Il est donc prévu - si besoin est - d'utiliser deux

\section{Vu schématique du modèle thermique 'LINES' de la boucle diphasique à pompage capillaire CPL du satel- lite STENTOR.}

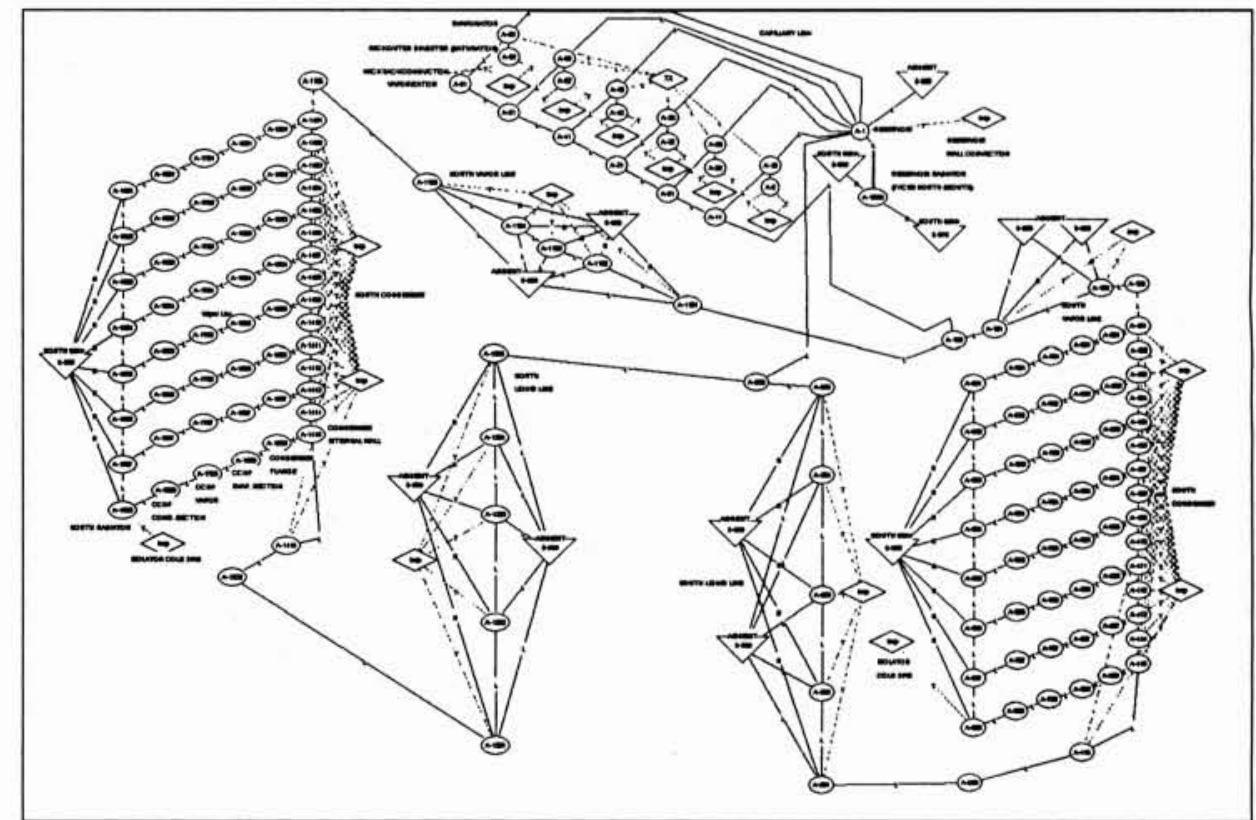




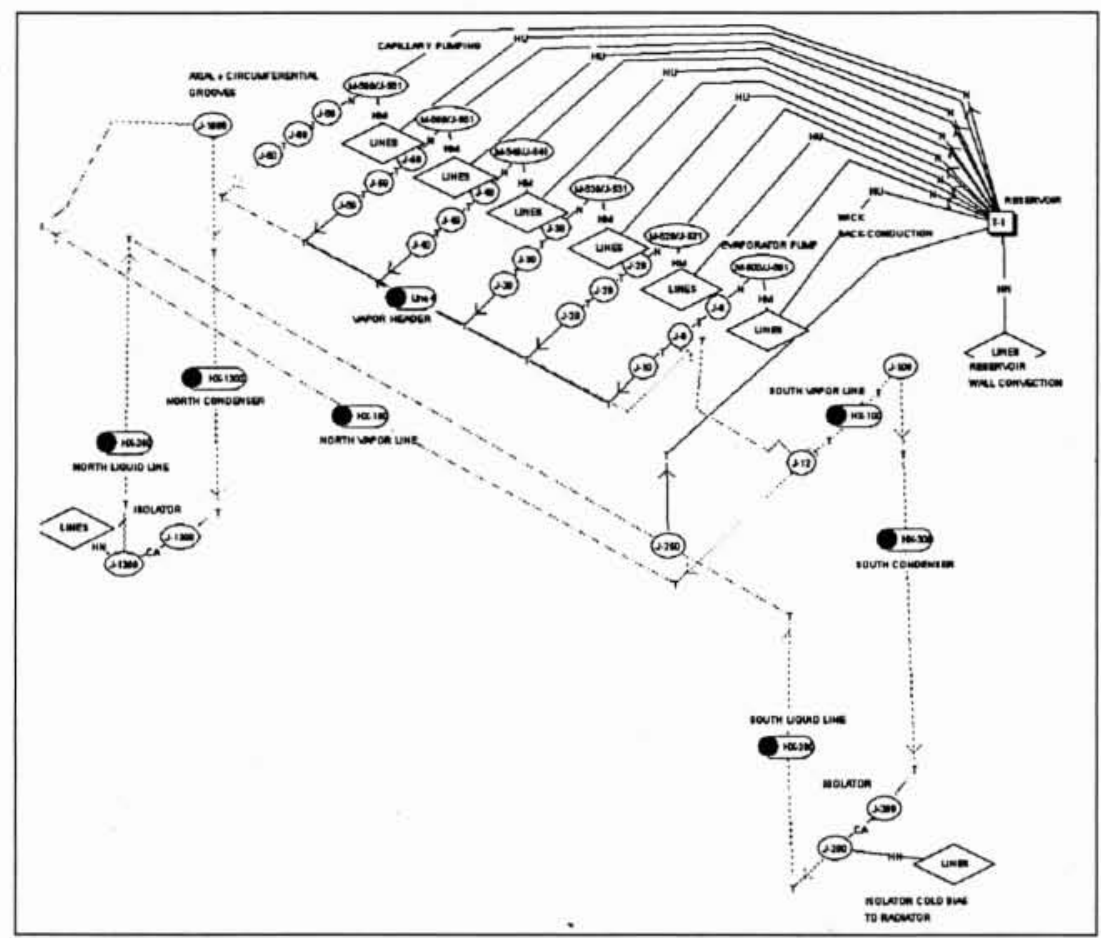

5. Vue schématique du modèle fluide 'LOOP' de la boucle diphasique à pompage capillaire CPL du satellite STENTOR.

nœuds fluides pour modéliser séparément la phase liquide et la phase vapeur. Les transferts de masse et de chaleur entre ces deux phases ne seront plus infinis. De ce fait, des efforts de modélisation supplémentaires seront nécessaires. Il faudra déterminer l'aire de l'interface entre les deux phases ainsi que les coefficients de transfert de chaleur liquide-interface et vapeur-interface. La caractérisation de ce type de coefficient de transfert est délicate même lorsqu'elle est menée à l'aide d'un code CFD tridimensionnel.

\section{- 3.2 Evaporateurs}

La paroi de chaque évaporateur est modélisée par un nœud thermique. Le gradient axial le long de l'évaporateur est donc supposé négligeable. La partie extérieure de la mèche capillaire de l'évaporateur, supposé en condition de saturation, est modélisée par un nœud thermique. L'action de pompage capillaire d'un évaporateur est simulée à l'aide du module CAPPMP du code SINDA/FLUINT. Ce module représente une sorte de 'surface capillaire' où le pompage est réalisé ou non par la mèche d'évaporateur. Il dépend en effet de la perte de pression totale subie par l'écoulement le long de la boucle en regard de la pression capillaire maximale que peut fournir la mèche d'évaporateur. La pompe est désamorcée si la contrainte en pression subie par la mèche d'évaporateur dépasse la pression capillaire maximale disponible calculée selon la relation de Laplace :

$$
\Delta \mathrm{p}_{\max \text { cap. }}=2 \sigma / \mathrm{r}_{\mathrm{c}}
$$

Un modèle d'évaporateur peut être construit sans, avec un ou plusieurs modules CAPPMP selon le degré de détail requis par le besoin d'analyse. Dans le modèle de la boucle CPL du satellite STENTOR, un module CAPPMP relie le fluide dans le réservoir et l'interface liquide-vapeur située à la partie extérieure de la mèche capillaire d'un évaporateur. Le liquide qui pénètre dans la mèche capillaire est légèrement sous-refroidi (relatif à la condition de saturation régnant à la partie extérieure de la mèche capillaire). L'échange de chaleur entre ce liquide et le matériau de la mèche crée un champ de température non-linéaire.

Le coefficient de transfert de chaleur d'un évaporateur dépend fortement des matériaux utilisés ainsi que de la méthode de construction (type d'interface entre mèche et cannelures). Le coefficient de transfert de chaleur entre la semelle d'évaporateur de la boucle CPL et la partie externe de la mèche capillaire est obtenu expérimentalement.

A la condition de saturation, la chaleur qui pénètre dans la mèche sert essentiellement à évaporer le liquide situé à la partie extérieure de la mèche capillaire. Le taux d'évaporation obéit à la relation suivante :

$$
\mathrm{m}_{\mathrm{v}}=\mathrm{Q} /\left(\mathrm{h}_{\mathrm{v}}-\mathrm{h}_{\mathrm{l}}\right)
$$

Une partie du chargement thermique se propage toutefois à travers la mèche capillaire par conduction pour provoquer une augmentation de la température du liquide dans le canal d'évaporateur ('wick back-conduction'). Bien entendu, ce chargement thermique parasite est plutôt faible lorsque le matériau poreux qui constitue la mèche est en matière plastique isolante (caractéristique de la plupart des CPL américaines). En revanche, il peut devenir relativement élevé dans le cas d'utilisation d'une mèche métallique frittée. Elle est employée en effet dans la fabrication des évaporateurs de la boucle CPL du satellite STENTOR. La modélisation précise du chargement thermique parasite est donc indispensable puisque celui-ci affecte le bilan thermique du réservoir surtout dans les cas froids. La quantité du chargement thermique parasite, qui pénètre l'évaporateur et qui se dirige vers le réservoir, dimensionne la taille du radiateur dédié au réservoir. Cette fuite thermique influe - dans une large mesure - sur le comportement des deux condenseurs de la boucle CPL. Pour estimer sa valeur, nous évaluons tout d'abord la conductance radiale à travers la mèche poreuse. Elle est estimée selon la formulation suivante : 


$$
\mathrm{UA}_{\mathrm{m}}=\left(2 \pi \mathrm{k}_{\mathrm{eff}} \mathrm{L}_{\mathrm{m}}\right) / \ln \left(\mathrm{D}_{\mathrm{m} \text { ext. }} / \mathrm{D}_{\mathrm{m} \text { int. }}\right)
$$

Le terme de la conductivité de la mèche capillaire 'mouillée' $\left(\mathrm{k}_{\mathrm{eff}}\right)$ est évalué de manière conservative selon la formulation parallèle classiquement utilisée pour les milieux poreux :

$$
\mathrm{k}_{\mathrm{eff}}=\varepsilon \mathrm{k}_{1}+(1-\varepsilon) \mathrm{k}_{\mathrm{m}}
$$

La valeur de cette conductivité est ensuite ajustée selon la température du liquide pénétrant le milieu poreux. Le coefficient de film entre la partie interne de la mèche et le fluide se trouvant dans le cour d'évaporateur est considéré dans un premier temps comme infini. Cette hypothèse est conservative au regard de la détermination du chargement thermique parasite.

Un lien capillaire entre évaporateur et réservoir caractérise le concept HPCPL (High Performance Capillary Pumped Loop) de SABCA. Il permet une alimentation ininterrompue d'un évaporateur par le liquide provenant du réservoir même lorsque le canal d'évaporateur est rempli de vapeur [4]. En l'absence des bulles de vapeur dans le cœur d'évaporateur ou plus globalement lorsque le liquide rentrant dans l'évaporateur est suffisamment sous-refroidi, le lien capillaire n'est pas opérationnel (pas de pompage capillaire).

Les cannelures de vapeur sont modélisées par les conducteurs fluides (TUBES). Le processus d'évaporation, qui a lieu à la partie extérieure de la mèche capillaire, injecte de la masse dans les cannelures suivant une direction perpendiculaire à celle de l'écoulement de vapeur le long des cannelures. Les différences en direction et en module entre les vecteurs de flux de quantité de mouvement créent un gradient de vitesse axiale. L'accélération de l'écoulement de vapeur dans les cannelures est prise en compte par le modèle.

Le présent modèle simule le fonctionnement de la boucle CPL en condition de vol autour de l'orbite géostationnaire. Il sera prochainement remanié en vue des efforts de corrélation des résultats du modèle de calcul aux résultats d'essais au laboratoire. Au sol, trois types de mode de fonctionnement doivent être analysés. En mettant tous les composants d'une boucle à pompage capillaire en position horizontale, l'influence de la gravité est minimisée (mode horizontal). En mode dit 'hauteur adverse', l'évaporateur est placé au-dessus du condenseur. Enfin, en mode de reflux, l'évaporateur est situé au-dessous du condenseur. Sous cette dernière configuration, les forces de volume assistent la circulation du fluide dans la boucle. La circulation du fluide résulte donc d'une action de type thermosiphon plutôt que d'un effet de capillarité.

\subsection{Condenseur}

Deux tubes identiques à rainures axiales internes - chacun des deux en contact avec une source froide - sont utilisés pour condenser la vapeur générée par les évaporateurs. Chaque condenseur est modélisé par 16 nœuds thermiques et 16 nœuds fluides. En toute première approche, nous traitons les condenseurs de la boucle CPL comme les condenseurs circulaires d'un diamètre égal au diamètre hydraulique du tube à rainures axiales employé. En modélisant une portion du fluide dans le condenseur à rainures axiales internes par un seul nœud fluide, nous ne faisons pas de distinction entre le liquide se trouvant dans les rainures axiales et la vapeur se trouvant dans le cœur du condenseur. Or, la présence des rainures axiales dans le condenseur a pour effet de piéger et de drainer le liquide, maintenant donc un taux de vide relativement constant le long du condenseur. Mais, le rayon de pompage capillaire des rainures axiales est relativement large donc la pression capillaire associée est plutôt faible. La différence de pression entre phases est faible devant le gradient de pression axial du condenseur. Les nœuds fluides séparés, pour les rainures et pour le cœur vapeur, n'ont donc pas été jugés nécessaires.

L'écoulement diphasique est supposé homogène. Cette hypothèse est en réalité adaptée pour les écoulements diphasiques à bulles dispersées ou à gouttelettes dispersées. Pour ces écoulements, les vitesses de glissement entre phases sont faibles. Rappelons que dans le modèle homogène on a l'égalité de vitesses du liquide et de la vapeur. L'écoulement diphasique se comporte comme un écoulement monophasique d'un fluide ayant des propriétés intermédiaires entre celles d'une pure phase vapeur et celles d'une pure phase liquide. Or, dans un écoulement diphasique co-courant liquide-vapeur comme celui observé dans les condenseurs CPL, la vapeur se déplace en général plus vite que le liquide. On peut donc s'attendre - pour un titre d'écoulement donné - à ce que le taux de vide réel soit inférieur à celui obtenu par le modèle homogène. Le taux de vide d'environ $60 \%$ correspond à la configuration d'une section du condenseur $\mathrm{CPL}$ où le liquide remplit toutes les rainures et la vapeur occupe tout le canal interne. Selon le modèle homogène, le titre correspondant est de $\mathrm{x}=0,02$. En appliquant les corrélations de Zivi, Lockhart \& Martinelli, Thom et Baroczy [5], les titres d'écoulement sont compris entre 0,03 et 0,08 . Nous nous contentons donc, à ce stade de l'étude, du modèle homogène pour l'estimation du taux de vide.

Les corrélations de perte de charge en écoulement diphasique qui sont utilisées dans le modèle sont celles recommandées par Crowley [6]. II s'agit d'un ensemble de modèles développés pour prédire les transitions entre les régimes d'écoulement diphasique dans les tubes, pour tout vecteur et module d'accélération allant de la microgravité à la gravité terrestre. Le régime d'écoulement est estimé en fonction des taux de vide, vitesses, direction et module des forces de volume, propriétés de fluide etc. Les quatre régimes d'écoulement considérés sont l'écoulement à bulles, l'écoulement intermittent à poches et à bouchons, l'écoulement annulaire et l'écoulement stratifié. Les méthodes contenues dans cette approche ont été jugées extrapolables aux autres fluides que de l'eau (l'ammoniac est le fluide utilisé dans la boucle CPL) et satisfaisantes pour les conditions de microgravité.

Il existe actuellement peu de corrélations de transfert de chaleur de condensation en film qui ont été développées pour inclure une grande variété de fluides et de régimes d'écoulement notamment en microgravité. La corrélation de Rohsenow/Traviss, interne au code SINDA/FLUINT, est actuellement utilisée pour le modèle du condenseur CPL. La corrélation de Rohsenow a été établie en particulier pour un écoulement convectif du régime annulaire. Ce régime est probablement prépondérant dans le processus de condensation dans le condenseur CPL. En effet, les rainures axiales favorisent l'établissement du régime annulaire de l'écoulement diphasique dans le condenseur, notamment en microgravité. L'application de la corrélation de Rohsenow pour un tube particulier à rainures axiales (en prenant son diamètre hydraulique) et pour un autre couple fluide-solide (aluminium-ammoniac), qui ne sont pas ceux à partir desquels la 
corrélation était établie, est certes sujette à caution. Comme l'est d'ailleurs, l'application de toute autre corrélation disponible dans la littérature qui n'a pas été établie sous les mêmes configuration géométrique et fonctionnement du condenseur CPL.

Les coefficients de transfert de chaleur estimés par la corrélation de Rohsenow décroissent lorsque la couche liquide s'épaissit. Or, dans un tube à rainures axiales, le liquide est redistribué de façon plutôt uniforme par les rainures. Nous devons donc tenir compte des effets de drainage du liquide par les rainures axiales. Les difficultés de modélisation résident donc dans cette prise en compte simultanée des deux phénomènes de condensation en film et condensation en rainures axiales. En l'absence des corrélations adaptées, une approche simple consiste à effectuer une moyenne arithmétique entre les prédictions données par Rohsenow et celles valables dans la section de condensation d'un caloduc à rainures axiales [7]. Il s'agit là d'un processus intermédiaire entre condensation en film liquide classique et condensation en rainures axiales. Enfin, la corrélation classique de DittusBoelter prédit l'échange convectif monophasique dans la zone inactive du condenseur où s'effectue le sous-refroidissement du liquide.

\section{• 3.4 Modèle d'isolateur}

Les deux isolateurs des deux condenseurs CPL, munis d'une structure capillaire, ont pour rôle de bloquer la progression de la vapeur non encore condensée vers la ligne de transport de liquide de la boucle CPL. Ils servent par conséquent de régulateur aux écoulements dans les deux condenseurs. Ces deux isolateurs sont représentés chacun par un conducteur fluide CAPIL du code SINDA/FLUINT. Cet élément est muni d'une fonctionnalité dite 'phase-specific suction' qui permet d'extraire soit la phase liquide soit la phase vapeur d'un volume de contrôle (nœud fluide) situé en amont de l'élément CAPIL. Lorsque l'écoulement diphasique pénètre l'isolateur, la résistance à l'écoulement est évaluée par la relation de Laplace. Si l'écoulement pénétrant l'isolateur est monophasique liquide, la conductance de l'écoulement capillaire est estimée par la relation :

$$
\mathrm{CFC}=\left(2 \pi \kappa \mathrm{L}_{\mathrm{m}}\right) / \ln \left(\mathrm{D}_{\mathrm{m} \text { ext. }} / \mathrm{D}_{\mathrm{m} \text { int. }}\right)
$$

\section{- 3.5 Modèle des lignes de transport de fluide}

Les lignes de vapeur et de liquide sont modélisées par les nœuds thermiques et les nœuds fluides. L'isolation des lignes de transport du liquide et de la vapeur est modélisée. L'échange de chaleur entre la paroi de la ligne liquide et la température ambiante peut influer fortement sur le niveau de sous-refroidissement du liquide entrant dans l'évaporateur. Une conductance de chaleur plus élevée entre la ligne de transport de liquide et l'environnement résultera en une perte du niveau de sous-refroidissement. L'isolation de la ligne liquide constitue donc un élément critique dans le fonctionnement de la boucle CPL.

\section{IV — QUELQUES RÉSULTATS DE MODÉLISATION}

Nous nous préoccupons dans cet article des difficultés de caractérisation de l'écoulement diphasique rencontrées dans l'élaboration du modèle de la boucle CPL du satellite STENTOR. Par conséquent, sans rentrer dans les détails, nous pré- sentons dans la figure 6 un exemple d'évolution de température des composants de la boucle CPL selon une variation du niveau de chargement thermique appliqué sur les évaporateurs sous un scénario de vol orbital donné. La sensibilité des résultats du modèle à la valeur du chargement thermique parasite est présentée sur la figure 7 . En bref, nous observons ici l'importance de la détermination du chargement thermique parasite sur le niveau de sous-refroidissement du liquide dans un des deux condenseurs, en l'occurrence, le condenseur Nord (voir l'évolution de T1200).

\section{CONCLUSIONS ET PERSPECTIVES}

Un modèle thermohydraulique monodimensionnel de la boucle diphasique CPL multi-évaporateur à pompage capillaire du satellite STENTOR a été élaboré à l'aide du code SINDA/FLUINT. Quelques difficultés de caractérisation des composants de la boucle CPL ont été constatées durant la construction des modèles de composant. La complexité de la configuration du réservoir rend délicate la prédiction en vol orbital et au sol du coefficient de film. En déséquilibre thermodynamique, les transferts finis de chaleur et de masse entre les deux phases dans le réservoir doivent être caractéri-

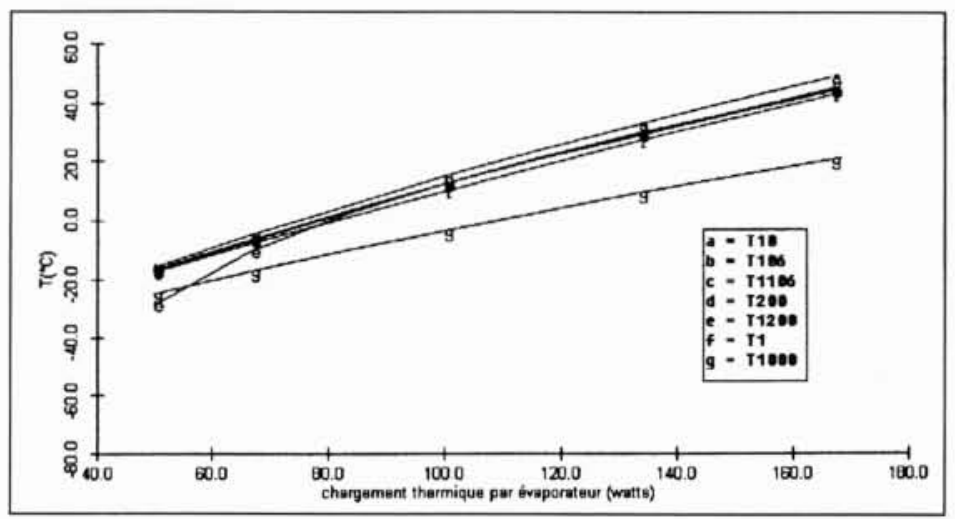

6. Evolution de température de quelques composants de la boucle CPL ; 1 : réservoir, 10 : évaporateur, 106 : entrée condenseur SUD, 200 : sortie condenseur SUD, 1106 : entrée condenseur Nord ; 1200 sortie condenseur Nord, 1000 : radiateur du réservoir.

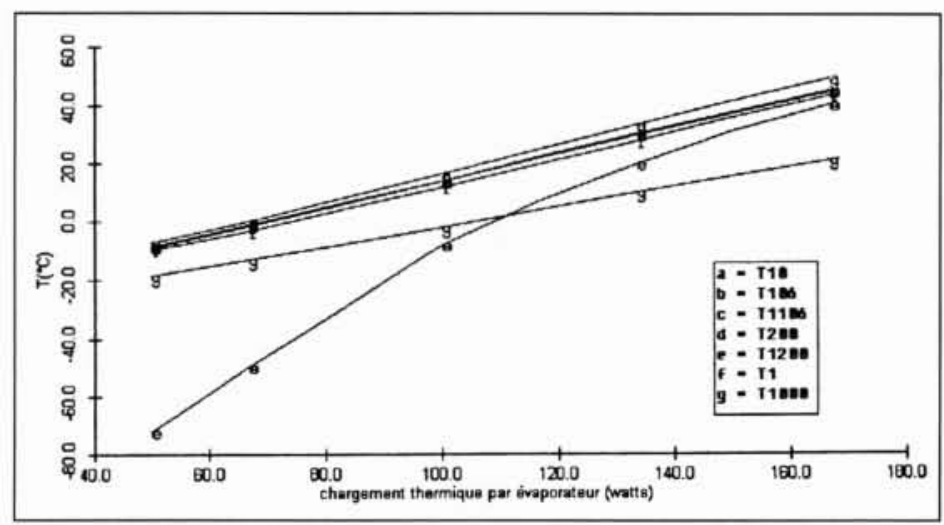

7. Evolution de température de quelques composants de la boucle CPL ; mèche capillaire 10 fois plus conductrice ; 1 : réservoir, 10 : évaporateur, 106 : entrée condenseur SUD, 200 : sortie condenseur SUD, 1106 : entrée condenseur Nord ; 1200 sortie condenseur Nord, 1000 : radiateur du réservoir. 
sés. En ce qui concerne les modèles d'évaporateur et d'isolateur, les difficultés sont liées notamment au caractère profondément tridimensionnel du milieu poreux utilisé comme mèche capillaire. Des corrélations de perte de charge et de transfert de chaleur par condensation existant dans la littérature ne sont pas toujours adaptées à la structure particulière des condenseurs CPL qui possèdent des rainures axiales internes. Enfin, le phénomène de microgravité ajoute un degré de difficulté supplémentaire à ces efforts de modélisation des composants de la boucle CPL.

En prenant quelques hypothèses simplificatrices, une version préliminaire du modèle thermohydraulique de la boucle CPL a été construite. Elle nous a permis - au niveau système - d'évaluer le comportement global de la boucle. Les validations expérimentales sont bien-entendu requises en vue de confirmer ou infirmer certaines hypothèses sur les modèles de composants.

\section{Références}

[1] Tuptahardia, T., Amidiev, M., Moschetr, B., Comparative analysis of test results of a two-phase loop using several software codes, Capillary Pumped Two-Phase Loops 'CPL 96' International Workshop, 8-10 May 1996.

[2] CuUnmore, B.A., Rivg, S.G., GoBLE, R.G., JeNSEN, C. L., SINDA FLUINT - Systems Improved Numerical Differencing Analyzer and Fluid Integrator User's Manual Version 3.2, Cullimore and Ring Technologies Inc., 1996

[3] RohSENOW, W.M., HARTNETT, J.P., Handbook of Heat Transfer. McGraw-Hill, 1973

[4] VAN Oost, S. et al., Test Results of reliable and Very High Capillary Multi-evaporators / Condenser Loop, SAE 951506, 25th International Conference on Environmental Systems, 1995

[5] CAREY, V. P., Liquid-vapor phase-change phenomena, Hemisphere Publishing corporation, 1992

[6] Crowley, C. J., Unified Flow Regime Predictions at Earth Gravity and Micnogravity, AIAA/ASME Thermophysics and Heat Transfer Conference, 1990

[7] Kamotani, Y., Analysis of Axially Grooved Heat Pipe Condensers, AIAA Paper 76-147, 1976

\begin{tabular}{|c|c|c|c|c|c|}
\hline \multicolumn{6}{|c|}{ NOMENCLATURE } \\
\hline$\Delta \mathrm{p}_{\max \text { cap. }}$ & : & pression capillaire maximale de la mèche & $\mathrm{L}_{\mathrm{m}}$ & : & longueur de la mèche \\
\hline & : & tension superficielle du fluide & $\mathrm{D}_{\mathrm{m} \text { ext. }}$ & : & diamètre extérieur de la mèche \\
\hline$r_{c}$ & : & rayon de courbure du ménisque & $\mathrm{D}_{\mathrm{m} \text { int. }}$ & : & diamètre intérieur de la mèche \\
\hline $\mathrm{m}_{\mathrm{v}}$ & : & taux d'évaporation du liquide & $\mathrm{k}_{1}$ & : & conductivité de la phase liquide \\
\hline Q & : & chargement thermique & $\varepsilon$ & : & porosité de la mèche \\
\hline $\mathrm{h}_{\mathrm{v}}$ & : & enthalpie de la phase vapeur & $\mathrm{k}_{\mathrm{m}}$ & : & conductivité de la mèche sèche \\
\hline$h_{1}$ & : & enthalpie de la phase liquide & CFC & : & conductance de l'écoulement capillaire \\
\hline $\mathrm{UA}_{\mathrm{m}}$ & : & conductance radiale de la mèche & $\kappa$ & : & perméabilité de la mèche \\
\hline $\mathrm{k}_{\mathrm{eff}}$ & : & $\begin{array}{l}\text { conductivité effective/composite de la } \\
\text { phase solide (mèche sèche) et du fluide }\end{array}$ & & & \\
\hline
\end{tabular}

